\title{
Impact of $\mathrm{CCl}$ on Performance Analysis of Downlink Satellite-Terrestrial Systems: Outage Probability and Ergodic Capacity Perspective
}

\section{Nhat-Tien Nguyen}

VSB-Technical University of Ostrava Faculty of Electrical Engineering and Computer Science: Vysoka Skola Banska-Technicka Univerzita Ostrava Fakulta Elektrotechniky a Informatiky

Hong-Nhu Nguyen

VSB-Technical University of Ostrava Faculty of Electrical Engineering and Computer Science: Vysoka Skola Banska-Technicka Univerzita Ostrava Fakulta Elektrotechniky a Informatiky

Anh-Tu Le

Industrial University of Ho Chi Minh City

Nhan Duc Nguyen ( $\nabla$ nhan.nd@vlu.edu.vn )

Industrial University of Ho Chi Minh City https://orcid.org/0000-0002-1561-7914

Dinh-Thuan Do

Asia University

MIROSLAV VOZNAK

VSB-Technical University of Ostrava Faculty of Electrical Engineering and Computer Science: Vysoka Skola Banska-Technicka Univerzita Ostrava Fakulta Elektrotechniky a Informatiky

\section{Research}

Keywords: non-orthogonal multiple access, satellite-terrestrial systems, outage probability, co-channel interference

Posted Date: November 22nd, 2021

DOI: https://doi.org/10.21203/rs.3.rs-1067017/v1

License: (c) (i) This work is licensed under a Creative Commons Attribution 4.0 International License.

Read Full License 


\title{
Impact of CCI on Performance Analysis of Downlink Satellite-Terrestrial Systems: Outage Probability and Ergodic Capacity Perspective
}

\author{
Nhat-Tien Nguyen ${ }^{1} \cdot$ Hong-Nhu \\ Nguyen $^{1} \cdot$ Anh-Tu Le ${ }^{2}$. Nhan Duc \\ Nguyen $^{3, \star}$. Dinh-Thuan Do ${ }^{4}$. Miroslav \\ Voznak $^{1}$
}

the date of receipt and acceptance should be inserted later

\begin{abstract}
The evolution of non-orthogonal multiple access (NOMA) has raised many opportunities for massive connectivity with less latency in signal transmissions at great distances. Power-Domain NOMA transmits user signals superimposed in the same resource block by varying the power coefficient of each user according to their channel state information (CSI). At the receiver's end, successive interference cancellation (SIC) is performed to extract the desired signal from the superimposed signal. Imperfect CSI should therefore be studied in this context. Satellite-terrestrial networks and relay networks have already gained significance in the field of communications through their efficient data transmission techniques. We aimed to integrate NOMA with a satellite communications network under both imperfect CSI and co-channel interference (CCI) from nearby systems with respect to analysis of ground user performance. In our considered system, two users perform downlink communications under Power-Domain NOMA. We analyzed the performance of this system with two modes of shadowing effect: Heavy Shadowing (HS) and Average Shadowing (AS). Performance was analyzed in terms of the outage probability and ergodic capacity of the system. We derived closed-form expressions and performed a numerical analysis. We discovered that the performance of two destinations depends on the strength of the transmit power at the satellite. However, floor outage occurs because the system depends on other parameters, such as satellite link modes, noise levels, and the number of interference sources. More specifically, if, for example, the number of interference sources is 5 , the outage performance of the system experiences a decrease of approximately $40 \%$ at a signal to noise ratio (SNR) of $30 \mathrm{~dB}$ at the satellite. Outage probability and ergodic capacity became saturated at SNRs of $50 \mathrm{~dB}$ and $45 \mathrm{~dB}$, respectively. To verify the authenticity of the derived closed-form expressions, we also performed Monte-Carlo simulations.
\end{abstract}

\footnotetext{
* Corresponding author. E-mail: nhan.nd@vlu.edu.vn
} 
Keywords non-orthogonal multiple access · satellite-terrestrial systems · outage probability $\cdot$ co-channel interference

\section{Introduction}

Satellite communications are especially able to provide radio access in difficult areas [1]. Because satellite networks can provide higher quality of services (QoS) for comparatively less cost, they can also attain significant improvements in the efficiency of fixed and mobile satellite services. In the evolution of fifth generation $(5 \mathrm{G})$ networks, satellite communications have been viewed as a potential addition to many technologies such as the Internet of Things (IoT), sensor networks and relaying communications [2]. The future of satellite networks is expected to support services of massive connectivity and reduce operational costs. Therefore, they can be deployed through their integration with various geostationary and non-geo-stationary orbital satellites by applying cooperative transmission or cognitive radio networks to increase the spectrum efficiency. To date, most satellite networks have adopted the orthogonal multiple access (OMA) technique for the transmission and reception of data [3].

The major disadvantage of OMA is that it cannot meet the growing requirements of communications networks. Under OMA, efficient spectrum use and limitations on the number of users have become major challenges which diminish system performance. In the present paper, we consider non-orthogonal multiple access (NOMA) to tackle the challenges raised by OMA. Of the two NOMA categories, we applied Power-Domain NOMA since much of the research has proved this system as having promising features. In NOMA, signals are transmitted superimposed in the same resource block by varying the power level of each user according to their channel gain. To identify the required user signal at the receiver, the system applies successive interference cancellation (SIC) and thereby extracts the required signal. As mentioned, NOMA uses the same resource block for multiple users and thus increases the efficiency of spectrum use at a reasonable level of implemented complexity [4], [5]. The NOMA technique has achieved significant attention from researchers around the globe and is a promising technology with advantages which can be exploited in $5 \mathrm{G}$ communications. Numerous studies have been performed to compare the performance of the NOMA system to OMA. The main finding is that NOMA is efficient [6], [7]. With an increase in spectrum efficiency, the benefits of NOMA performance can be more prevalent if NOMA is integrated with other techniques.

Various studies have introduced the NOMA technique in satellite communications [8]- [16]. In [11], the authors studied integration of the NOMA technique with multi-beam satellite networks, while in [12], the authors investigated integration of NOMA with cognitive satellite networks to increase ergodic performance of the system. The performance of NOMA-hybrid satellite relay networks (HSRN) was studied in [13] [14]. NOMA integrated cognitive HSRN has been studied to analyze outage performance [15]. The performance 
of a similar system studied in [13] was investigated with the effect of hardware impairments [16]. The work in [17]- [19] considered NOMA-based satelliteterrestrial networks to increase the efficiency of the spectrum by beamforming. In [20], a cooperative NOMA-HSRN was considered in which the user with better channel gain acted as a relay to the remaining users in the cluster. In [21], the authors studied the effect of imperfect channel state information (CSI) and channel impairments (CI) in a NOMA-based terrestrial mobile communications network (TMCN) which functioned with multiple relays. In [22], the authors considered NOMA-based integrated terrestrial satellite networks (ISTN) to study the effect of relaying configurations such as Amplify and Forward (AF) and Decode and Forward (DF). The authors in [28] investigated a system similar to the study in [22] and explored the effect of CI under a DF relay configuration.

In the context of NOMA-HSRN, the effect of co-channel interference (CCI) in all system models has rarely been addressed. In practice, NOMA-HSRN might experience a rich CCI situation, which is an important consideration in the deployment of NOMA and HSRN in wireless communications. It can be demonstrated that the aggressive reuse of spectrum resources leads to degraded performance because of the effect of CCI. As a result, it is more than simply an important priority consideration, as the performance of NOMAHSRN is guaranteed only if CCI is taken into account. To the best of the authors' knowledge, the performance of NOMA-HSRN under the impact of CCI has not been solved yet. Aiming to overcome the effect of CCI, which is unavoidable in practical scenarios, the authors in [23] reported degraded performance in a single-user hybrid satellite-terrestrial amplify-and-forward relay network (HSTAFRN) with multiple Rayleigh-faded interference sources. The performance metrics of a downlink multi-user HSTAFRN were examined using a fixed-gain relaying protocol under the effect of CCI [24]. The authors in [25] employed dual-hop relay networks which assumed interference-limited relays and noisy destinations. These may arise from cell-edge or frequency-division relaying [26], although the NOMA-HSRN still experiences worse performance under the effect of CCI. The authors in [27] deployed a single-antenna satellite for a multi-user HSTAFRN system and evaluated its outage performance under the effect of both CCI and outdated CSI.

Satellite networks have been designed to replace terrestrial communications systems, but challenges still exist in some aspects of signal processing. The referred studies suggest that integrating NOMA with satellite systems will extend the efficiency of communication between users. Although satellite systems have numerous advantages, challenges such as fading effect and interference require solutions. The majority of research has studied the effect of various scenarios, including imperfect SIC, imperfect CSI and channel impairments, but none has mentioned the effect on system performance from CCI. Therefore, in the present paper, we studied the effect of interference by considering shadowing and interference links in dual-user communications occurring under a NOMA-assisted satellite network. We studied the shadowing effect in two modes: Heavy shadowing (HS) and Average Shadowing (AS). We 
also investigated the effect of interference links on communications channels in HS mode. In contrast to similar studies listed in Table 1 and to highlight the superiority of NOMA-aided satellite systems, performance was analyzed completely in terms of outage probability (OP) and ergodic capacity (EC).

The primary contributions of the paper are manifold:

- In contrast to other studies, the present paper presents a complex NOMAbased terrestrial satellite relay network with two users on the ground and a relay which encounters interference from nearby sources. We provide system performance metrics by considering a Shadowed-Rician channel between the satellite and relay, and a Nakagami- $m$ channel between the relay and destination.

- The system encounters HS and AS effects as a result of the shadowing channel links. We analyzed and compared the system's performance under these two shadowing modes.

- We derived the closed-form expressions for outage probability and ergodic capacity of both users in a dedicated NOMA user group. A performance gap is expected for these users depending on their demands and the portion of power allocated to each user.

- Finally, we performed a numerical analysis and Monte-Carlo simulations for the derived expressions to verify their authenticity and analyze the system's performance. We illustrate the system's performance in HS mode by varying the interference links and mean square error of the channels.

The paper is organized as follows. Section II explains the system model and types of signal received from the users. Section III provides a performance analysis and describes the mathematical expressions for outage probability, ergodic capacity and diversity order of the system. Section IV provides an analysis and simulations of the expressions obtained in Section III. Section V concludes the paper with the attained results.

\section{Method}

In this section, we assume a satellite (S), a relay (R) and two users $D_{i}, i \in$ $(1,2)$. All nodes are equipped with a single antenna, and the relay operates with the DF protocol. The relay node is also affected by $N$ co-channel interference sources $\left\{I_{n}\right\}_{n=1}^{N}$. The link from $\mathrm{S}$ to $D_{i}$ is not available because of heavy shadowing [29]. $h_{R}$ denotes the channel coefficient from $\mathrm{S}$ to $\mathrm{R}$ and follow a Shadowed-Rician channel, $h_{i}$ denotes the channel coefficients from $\mathrm{R}$ to $D_{i}$ and follow a Nakagami- $m$ channel, $h_{n R}$ denotes the channel coefficient of the link between the $n$-th interference source and relay and follows independent and non-identically distributed (i.ni.d.) Nakagami- $m$ random variable (RVs). Under these conditions, the CSI procedure exhibits error. The estimation channel is expressed as [30]

$$
h_{j}=\hat{h}_{j}+e_{j},
$$

where $j \in\{R, 1,2\}, e_{j}$ is the error term for $C N\left(0, \sigma_{e_{j}}^{2}\right)[31]$. 
Table 1 Summary of related works.

\begin{tabular}{|c|c|c|c|}
\hline References & $\begin{array}{l}\text { Contexts built on } \\
\text { NOMA }\end{array}$ & $\begin{array}{l}\begin{array}{l}\text { Performance Anal- } \\
\text { ysis }\end{array} \\
\end{array}$ & Contributions \\
\hline$[11]$ & $\begin{array}{l}\text { Multi-beam satellite } \\
\text { system }\end{array}$ & Data rate fairness & $\begin{array}{l}\text { Performance comparison of SIC to simultaneous non- } \\
\text { unique detection (SND) }\end{array}$ \\
\hline$[12]$ & $\begin{array}{lr}\text { Cognitive } & \text { hybrid } \\
\text { satellite } & \text { terrestrial } \\
\text { networks } & \end{array}$ & Ergodic capacity & $\begin{array}{l}\text { Investigated improvement of the radio spectrum effi- } \\
\text { ciency }\end{array}$ \\
\hline$[13],[15]$ & $\begin{array}{l}\text { Hybrid satellite ter- } \\
\text { restrial networks }\end{array}$ & Outage performance & $\begin{array}{l}\text { Investigated power allocation and user fairness prob- } \\
\text { lems }\end{array}$ \\
\hline$[16]$ & $\begin{array}{l}\text { Integrated satellite } \\
\text { terrestrial networks }\end{array}$ & $\begin{array}{l}\text { Outage performance } \\
\text { and energy efficiency }\end{array}$ & $\begin{array}{l}\text { Investigated system performance under the effect of } \\
\text { hardware impairments }\end{array}$ \\
\hline$[18]$ & $\begin{array}{l}\text { Integrated satellite } \\
\text { terrestrial networks }\end{array}$ & System capacity & $\begin{array}{l}\text { Investigated beamforming for maximization of the } \\
\text { minimum channel correlation and achieved efficient } \\
\text { user pairing with the proposed scheme }\end{array}$ \\
\hline$[22]$ & $\begin{array}{lr}\text { Hybrid } & \text { satellite } \\
\text { terrestrial } & \text { relay } \\
\text { networks } & \end{array}$ & Outage performance & $\begin{array}{l}\text { Investigated the system under the effect of imperfect } \\
\text { CSI and analyzed the system with channel estimation } \\
\text { errors and fading parameters. Proposed a low complex- } \\
\text { ity algorithm to yield efficient results. }\end{array}$ \\
\hline Our research & $\begin{array}{l}\text { Terrestrial satellite } \\
\text { relay network with } \\
\text { CCI and imperfect } \\
\text { CSI }\end{array}$ & $\begin{array}{l}\text { Outage Performance } \\
\text { and ergodic capacity }\end{array}$ & $\begin{array}{l}\text { Investigated and compared performance with CCI and } \\
\text { imperfect CSI. }\end{array}$ \\
\hline
\end{tabular}

Table 2 Table of parameters.

\begin{tabular}{|l|l|}
\hline Notation & Definition \\
\hline$N$ & Co-channel interference sources affecting the relay. \\
\hline$x_{i}$ & Messages for destination $D_{i}$ \\
\hline$x_{n}$ & Unit energy signal of the $n$-th interference source \\
\hline$A_{i}$ & Power allocation coefficients, where $A_{1}+A_{2}=1$ and $A_{1}>A_{2} \cdot$ \\
\hline$P_{S}$ & Transmit power at $\mathrm{S}$ \\
\hline$P_{R}$ & Transmit power at $\mathrm{R}$ \\
\hline$P_{C n}$ & Transmit power of the $n$-th interference source \\
\hline$P_{C n}$ & Transmit power of the $n$-th interference source \\
\hline$n_{j}$ & Additive white Gaussian noise $($ AWGN $)$ for $C N\left(0, N_{0}\right)$ \\
\hline$h_{R}$ & Channel coefficient from $\mathrm{S}$ to R \\
\hline$h_{i}$ & Channel coefficient from $\mathrm{R}$ to $D_{i}$ \\
\hline$h_{n R}$ & Channel coefficient from $n$-th interference source to the relay \\
\hline$\hat{h}_{j}$ & Estimated channel coefficients \\
\hline$e_{j}$ & Channel estimation error \\
\hline$\Gamma()$. & Complete Gamma function \\
\hline$\Gamma(.,)$. & Upper incomplete Gamma function \\
\hline${ }_{1} F_{1}(., .,)$. & Confluent hypergeometric function of the first kind \\
\hline$G_{1,1}^{1,1}[]$. & Meijer's G-function \\
\hline$G_{1,[1,1], 0,[1,1]}^{1,1,1,1}[.]$. & Meijer's G-function with two variables \\
\hline
\end{tabular}


In the first phase, $\mathrm{S}$ transmits the signal $\sqrt{P_{S} A_{1}} x_{1}+\sqrt{P_{S} A_{2}} x_{2}$ to $\mathrm{R}$, where $P_{S}$ is the transmit power, $A_{1}$ and $A_{2}$ are power allocation such that $A_{1}+A_{2}=1$ and $A_{1}>A_{2}$ assumed under the NOMA scheme. Then, the signal received at $\mathrm{R}$ is given as

$$
y_{R}=\left(\sqrt{A_{1}} x_{1}+\sqrt{A_{2}} x_{2}\right) \sqrt{P_{S}}\left(\hat{h}_{j}+e_{j}\right)+\sum_{n=1}^{N} \sqrt{P_{c n}} h_{n R} x_{n}+n_{R},
$$

where $P_{C n}$ is the transmit power of the $n$-th interference source and $n_{R}$ is the additive white Gaussian noise (AWGN) at $\mathrm{R}$ for $C N\left(0, N_{0}\right)$. The signal to interference plus noise ratio (SINR) is then used to decode $x_{1}$ and given as

$$
\Gamma_{R \rightarrow x_{1}}=\frac{\rho_{S} A_{1}\left|\hat{h}_{R}\right|^{2}}{\rho_{S} A_{2}\left|\hat{h}_{R}\right|^{2}+\gamma_{C}+\rho_{S} \sigma_{e_{R}}^{2}+1},
$$

where $\rho_{S}=\frac{P_{S}}{N_{0}}$ is the transmit SNR, $\rho_{C n}=\frac{P_{C n}}{N_{0}}$ and $\gamma_{C}=\sum_{n=1}^{N} \rho_{C n}\left|h_{n R}\right|^{2}$. Then, the SINR decoded $x_{2}$ is given as

$$
\Gamma_{R \rightarrow x_{2}}=\frac{\rho_{S} A_{2}\left|\hat{h}_{R}\right|^{2}}{\gamma_{C}+\rho_{S} \sigma_{e_{R}}^{2}+1} .
$$

In the second phase, relay $\mathrm{R}$ forwards the signals to the ground users. The signal received at $D_{i}$ is given as

$$
y_{D_{i}}=\left(\sqrt{A_{1}} x_{1}+\sqrt{A_{2}} x_{2}\right) \sqrt{P_{R}}\left(\hat{h}_{i}+e_{i}\right)+n_{i},
$$

where $P_{R}$ is the transmit power at $\mathrm{R}$ and $n_{D_{i}}$ AWGN for $C N\left(0, N_{0}\right)$. The main parameters are listed in Table 2 . The SINR which decodes $x_{1}$ at $D_{1}$ is given as

$$
\Gamma_{D_{1} \rightarrow x_{1}}=\frac{\rho_{R} A_{1}\left|\hat{h}_{1}\right|^{2}}{\rho_{R} A_{2}\left|\hat{h}_{1}\right|^{2}+\rho_{R} \sigma_{e_{1}}^{2}+1},
$$

where $\rho_{R}=\frac{P_{R}}{N_{0}}$, the SINR which decodes signal $x_{1}$ at $D_{2}$ is given as

$$
\Gamma_{D_{2} \rightarrow x_{1}}=\frac{\rho_{R} A_{1}\left|\hat{h}_{2}\right|^{2}}{\rho_{R} A_{2}\left|\hat{h}_{2}\right|^{2}+\rho_{R} \sigma_{e_{2}}^{2}+1},
$$

Applying SIC, the SINR which decodes its own signal $x_{2}$ at $D_{2}$ is computed according to

$$
\Gamma_{D_{2} \rightarrow x_{2}}=\frac{\rho_{R} A_{2}\left|\hat{h}_{2}\right|^{2}}{\sigma_{e_{2}}^{2}+1} .
$$

For performance analysis, these SINRs provide important information which allows us to compute probabilities. 


\section{Performance Analysis}

In this section, we analyze the two main system metrics with the assumed channel models below.

\subsection{Channel model}

Following the results in [32], the probability density function (PDF) of $\left|\hat{h}_{R}\right|^{2}$ is formulated by

$$
f_{\left|\hat{h}_{R}\right|^{2}}(x)=\alpha_{R} e^{-\beta_{R} x} F_{1}\left(m_{R}, 1, \delta_{R} x\right),
$$

where $\alpha_{R}=\frac{\left(\frac{2 b_{R} m_{R}}{2 b_{R} m_{R}+\Omega_{R}}\right)^{m_{R}}}{2 b_{R}}, \beta_{R}=\left(2 b_{R}\right)^{-1}, \delta_{R}=\frac{\Omega_{R}}{2 b_{R}\left(2 b_{R} m_{R}+\Omega_{R}\right)}, m_{R}$ is the fading severity parameter, $2 b_{R}$ and $\Omega_{R}$ denote multipath components and the average power of light of sight (LOS), respectively, and ${ }_{1} F_{1}(., .,$.$) is the$ confluent hypergeometric function of the first kind [44, Eq. 9.210.1]. Using [33], we can rewrite the PDF of $\left|h_{R}\right|^{2}$ as

$$
f_{\left|\hat{h}_{R}\right|^{2}}(x)=\alpha_{R} \sum_{k=0}^{m_{R}-1} \xi(k) x^{k} e^{-\Xi_{R} x}
$$

where $\xi(k)=\frac{(-1)^{k}\left(1-m_{R}\right)_{k} \delta_{R}^{k}}{(k !)^{2}}, \Xi_{R}=\beta_{R}-\delta_{R}$ and $(.)_{x}$ denotes the Pochhammer symbol [44, p. xliii]. Based on [44, Eq.3.351.2], the the cumulative distribution function $(\mathrm{CDF})$ of $\left|\hat{h}_{R}\right|^{2}$ can be obtained as

$$
F_{\left|\hat{h}_{R}\right|^{2}}(x)=1-\alpha_{R} \sum_{k=0}^{m_{R}-1} \xi(k) \sum_{l=0}^{k} \frac{k ! x^{l} e^{-\Xi_{R} x}}{l !\left(\Xi_{R}\right)^{k-l+1}} .
$$

The PDF and CDF of $\left|h_{i}\right|^{2}$ are then respectively given as [34]

$$
f_{\left|\hat{h}_{i}\right|^{2}}(x)=\left(\frac{m_{i}}{\Omega_{i}}\right)^{m_{i}} \frac{x^{m_{i}-1} e^{-\left(\frac{m_{i}}{\Omega_{i}}\right) x}}{\Gamma\left(m_{i}\right)},
$$

and

$$
\begin{aligned}
& F_{\left|\hat{h}_{i}\right|^{2}}(x)=1-\frac{1}{\Gamma\left(m_{i}\right)} \Gamma\left(m_{i}, \frac{m_{i} x}{\Omega_{i}}\right) \\
& =1-\sum_{b_{i}=0}^{m_{i}-1} \frac{1}{b_{i} !}\left(\frac{m_{i} x}{\Omega_{i}}\right)^{+b_{i}} e^{-\left(\frac{m_{i}}{\Omega_{i}}\right) x}
\end{aligned}
$$

where $m_{i}$ and $\Omega_{i}$ are the fading severity parameter and the average power, respectively, and $\Gamma(.,$.$) is the upper incomplete gamma function [44].$ 
Moreover, the PDF of $\gamma_{C}$ is calculated with corresponding severity parameters $\left\{m_{C n}\right\}_{n}^{N}$ and average powers $\left\{\Omega_{C n}\right\}_{n}^{N}$. Therefore, as in [35], [36] and [37], we can express the PDF of $\gamma_{C}$ as

$$
f_{\gamma_{C}}(x)=\left(\frac{m_{I}}{\Omega_{I}}\right)^{m_{I}} \frac{x^{m_{I}-1}}{\Gamma\left(m_{I}\right)} e^{-\left(\frac{m_{I}}{\Omega_{I}}\right) x}
$$

where the parameters $m_{I}$ and $\Omega_{I}$ are obtained from moment based estimators. For this, we define $\Theta=\sum_{n=1}^{I}\left|h_{n R}\right|^{2}$, and without loss of generality, we assume no power control is used, i.e., $P_{C n}=P_{C}$ or $\rho_{C n}=\rho_{C}$. Then, we have $\Omega_{I}=\rho_{C} \Omega_{C}$, where $\Omega_{C}=E[\Theta]=\sum_{n=1}^{N} \Omega_{C n}$ and $m_{I}=\frac{\Omega_{C}^{2}}{E\left[\Theta^{2}\right]-\Omega_{C}^{2}}$. From this, the exact moments of $\Theta$ can be obtained as

$$
\begin{aligned}
& E\left[\Theta^{n}\right] \approx \sum_{n_{1}=0}^{n} \sum_{n_{2}=0}^{n_{1}} \ldots \sum_{n_{N-1}=0}^{n_{N-2}}\left(\begin{array}{c}
n \\
n_{1}
\end{array}\right)\left(\begin{array}{c}
n_{1} \\
n_{2}
\end{array}\right)\left(\begin{array}{c}
n_{N-2} \\
n_{N-1}
\end{array}\right) \\
& \times E\left[\left|h_{1 R}\right|^{2\left(n-n_{1}\right)}\right] E\left[\left|h_{1 R}\right|^{2\left(n_{1}-n_{2}\right)}\right] \cdots E\left[\left|h_{1 R}\right|^{2\left(n_{N-1}\right)}\right]
\end{aligned}
$$

where $E\left[\left|h_{i R}\right|^{n}\right]=\frac{\Gamma\left(m_{C n}+\frac{n}{2}\right)}{\Gamma\left(m_{C n}\right)}\left(\frac{m_{C n}}{\Omega_{C n}}\right)^{-\frac{n}{2}}$.

3.2 Outage probability of $D_{1}$

An outage event of $D_{1}$ is given when $R$ and $D_{1}$ cannot detect $x_{1}$ correctly. Then, the outage probability of $D_{1}$ is given as

$$
\begin{aligned}
& P_{D_{1}}=\operatorname{Pr}\left(\min \left(\Gamma_{R \rightarrow x_{1}}, \Gamma_{D_{1} \rightarrow x_{1}}\right)<\gamma_{1}\right) \\
& =1-\underbrace{\operatorname{Pr}\left(\Gamma_{R \rightarrow x_{1}}>\gamma_{1}\right)}_{B_{1}} \underbrace{\operatorname{Pr}\left(\Gamma_{D_{1} \rightarrow x_{1}}>\gamma_{1}\right)}_{B_{2}},
\end{aligned}
$$

where $\gamma_{i}=2^{2 R_{i}}-1$, and $R_{i}$ is the target rate.

Proposition 1: Here, the closed-form of $B_{1}$ is given as

$$
\begin{aligned}
& B_{1}=\alpha_{R} \sum_{k=0}^{m_{R}-1} \sum_{l=0}^{k} \sum_{p=0}^{l}\left(\begin{array}{l}
l \\
p
\end{array}\right) \frac{\xi(k) k ! \Gamma\left(m_{I}+p\right)}{l ! \Gamma\left(m_{I}\right)\left(\Xi_{R}\right)^{k-l+1}} e^{-\Xi_{R} \phi_{1}\left(\rho_{S} \sigma_{e_{R}}^{2}+1\right)} \\
& \times\left(\phi_{1}\left(\rho_{S} \sigma_{e_{R}}^{2}+1\right)\right)^{l}\left(1+\frac{\Omega_{I} \Xi_{R} \phi_{1}}{m_{I}}\right)^{-m_{I}-p}\left(\frac{\Omega_{I}}{m_{I}\left(\rho_{S} \sigma_{e_{R}}^{2}+1\right)}\right)^{p}
\end{aligned}
$$

Proof: See Appendix A 
Next, using (6), $B_{2}$ is rewritten as

$$
\begin{aligned}
& B_{2}=\operatorname{Pr}\left(\frac{\rho_{R} A_{1}\left|\hat{h}_{1}\right|^{2}}{\rho_{R} A_{2}\left|\hat{h}_{1}\right|^{2}+\rho_{R} \sigma_{e_{1}}^{2}+1}>\gamma_{1}\right) \\
& =\operatorname{Pr}\left(\left|\hat{h}_{1}\right|^{2}>\phi_{2}\right) \\
& =1-F_{\left|\hat{h}_{1}\right|^{2}}\left(\phi_{2}\right)
\end{aligned}
$$

where $\phi_{2}=\frac{\left(\rho_{R} \sigma_{e_{1}}^{2}+1\right) \gamma_{1}}{\left(A_{1}-A_{2} \gamma_{1}\right) \rho_{R}}$. Based on the CDF of $\hat{h}_{i}$ in (13), $B_{2}$ can be expressed as

$$
B_{2}=\sum_{b_{1}=0}^{m_{1}-1} \frac{e^{-\frac{m_{1} \phi_{2}}{\Omega_{1}}}}{b_{1} !}\left(\frac{m_{1} \phi_{2}}{\Omega_{1}}\right)^{b_{1}} .
$$

Finally, substituting (17) and (19) into (16), $P_{D_{1}}$ can be obtained by

$$
\begin{aligned}
& P_{D_{1}}=1-\alpha_{R} \sum_{k=0}^{m_{R}-1} \sum_{l=0}^{k} \sum_{p=0}^{l} \sum_{b_{1}=0}^{m_{1}-1}\left(\begin{array}{l}
l \\
p
\end{array}\right) \frac{\xi(k) k ! \Gamma\left(m_{I}+p\right) e^{-\Xi_{R} \phi_{1}\left(\rho_{S} \sigma_{e_{R}}^{2}+1\right)-\frac{m_{1} \phi_{2}}{\Omega_{1}}}}{b_{1} ! l ! \Gamma\left(m_{I}\right)\left(\Xi_{R}\right)^{k-l+1}\left(\phi_{1}\left(\rho_{S} \sigma_{e_{R}}^{2}+1\right)\right)^{-l}} \\
& \times\left(\frac{m_{1} \phi_{2}}{\Omega_{1}}\right)^{b_{1}}\left(1+\frac{\Omega_{I} \Xi_{R} \phi_{1}}{m_{I}}\right)^{-m_{I}-p}\left(\frac{\Omega_{I}}{m_{I}\left(\rho_{S} \sigma_{e_{R}}^{2}+1\right)}\right)^{p}
\end{aligned}
$$

\subsection{Outage probability of $D_{2}$}

The outage events of $D_{2}$ occurs when $R$ and $D_{2}$ cannot detect $x_{2}$ correctly. Therefore, the outage probability of $D_{2}$ is given as

$$
\begin{aligned}
& P_{D_{2}}=\operatorname{Pr}\left(\min \left(\Gamma_{R \rightarrow x_{2}}, \Gamma_{D_{2} \rightarrow x_{2}}\right)<\gamma_{2}\right) \\
& =1-\operatorname{Pr}\left(\Gamma_{R \rightarrow x_{2}}>\gamma_{2}\right) \operatorname{Pr}\left(\Gamma_{D_{2} \rightarrow x_{2}}>\gamma_{2}\right)
\end{aligned}
$$

Proposition 2: The closed-form outage probability of $P_{D_{2}}$ is obtained as

$$
\begin{aligned}
& P_{D_{2}}=1-\alpha_{R} \sum_{k=0}^{m_{R}-1} \sum_{l=0}^{k} \sum_{p=0}^{l} \sum_{b_{2}=0}^{m_{2}-1}\left(\begin{array}{l}
l \\
p
\end{array}\right) \frac{k ! \xi(k) \Gamma\left(m_{I}+p\right) e^{-\Xi_{R} \psi_{1}\left(\rho_{S} \sigma_{e_{R}}^{2}+1\right)-\frac{m_{2} \psi_{2}}{\Omega_{2}}}}{b_{2} ! l ! \Gamma\left(m_{I}\right)\left(\Xi_{R}\right)^{k-l+1}\left(\psi_{1}\left(\rho_{S} \sigma_{e_{R}}^{2}+1\right)\right)^{-l}} \\
& \times\left(\frac{m_{2} \psi_{2}}{\Omega_{2}}\right)^{b_{2}}\left(1+\frac{\Omega_{I} \Xi_{R} \psi_{1}}{m_{I}}\right)^{-m_{I}-p}\left(\frac{\Omega_{I}}{m_{I}\left(\rho_{S} \sigma_{e_{R}}^{2}+1\right)}\right)^{p}
\end{aligned}
$$

Proof: See Appendix B 
3.4 Diversity order

To gain some insight, we derive under the asymptotic outage probability of $D_{i}$ under a high SNR $\left(\rho=\rho_{S}=\rho_{R} \rightarrow \infty\right)$. The diversity order is defined as [38]

$$
d=-\lim _{\rho \rightarrow \infty} \frac{\log \left(P_{D_{i}}^{\infty}\right)}{\log (\rho)}
$$

where $P_{D_{i}}^{\infty}$ is the asymptotic outage probability of $D_{i}$.

Proposition 3: The asymptotic outage probability of $D_{1}$ is given as

$$
\begin{aligned}
& P_{D_{1}}^{\infty}=1-\left(1-\frac{1}{\Gamma\left(m_{1}+1\right)}\left(\frac{m_{1} \phi_{2}}{\Omega_{1}}\right)^{m_{1}}\right) \\
& \times\left(1-\alpha_{R} \phi_{1}\left(\frac{\left(m_{I}\right) !}{\Gamma\left(m_{I}\right)}\left(\frac{\Omega_{I}}{m_{I}}\right)+\left(\rho_{S} \sigma_{e_{R}}^{2}+1\right)\right)\right)
\end{aligned}
$$

Proof: See Appendix C

Similarly, the asymptotic of $D_{2}$ can be expressed by

$$
\begin{aligned}
& P_{D_{2}}^{\infty}=1-\left(1-\frac{1}{\Gamma\left(m_{2}-1\right)}\left(\frac{m_{2} \psi_{2}}{\Omega_{2}}\right)^{m_{2}}\right) \\
& \left(1-\alpha_{R} \psi_{1}\left(\frac{\left(m_{I}\right) !}{\Gamma\left(m_{I}\right)}\left(\frac{\Omega_{I}}{m_{I}}\right)+\left(\rho_{S} \sigma_{e_{R}}^{2}+1\right)\right)\right)
\end{aligned}
$$

The results in (24) and (25) refer to limits of outage performance in the region of high SNR. It can be predicted that the outage performance of two ground users encounters the lower bound even though we improve other system parameters. As discussed, the diversity is then zero.

3.5 Ergodic Capacity of $D_{1}$

The ergodic capacity of $x_{i}$ is expressed as [39]

$$
R_{x_{1}}=\frac{1}{2 \log (2)} \int_{0}^{\frac{A_{1}}{A_{2}}} \frac{1-F_{Q_{1}}(x)}{1+x} d x
$$

where $Q_{1}=\min \left(\Gamma_{R \rightarrow x_{1}}, \Gamma_{D_{1} \rightarrow x_{1}}\right)$. 
Proposition 4: The closed-form ergodic capacity of $x_{1}$ is given as (27), where $\Psi_{1}=\frac{\left(\rho_{S} \sigma_{e_{R}}^{2}+1\right) \Xi_{R}\left(1+\theta_{p}\right)}{A_{2} \rho_{S}\left(1-\theta_{p}\right)}+\frac{m_{1}\left(\rho_{R} \sigma_{e_{1}}^{2}+1\right)\left(1+\theta_{p}\right)}{A_{2} \Omega_{1} \rho_{R}\left(1-\theta_{p}\right)}$.

$$
\begin{aligned}
& R_{x_{1}} \approx \frac{\alpha_{R}}{2 \ln (2)} \sum_{k=0}^{m_{R}-1} \sum_{l=0}^{k} \sum_{p=0}^{l} \sum_{b_{1}=0}^{m_{1}-1}\left(\begin{array}{c}
l \\
p
\end{array}\right) \frac{\xi(k) k !}{b_{1} ! l ! \Gamma\left(m_{I}\right)} \frac{\Gamma\left(m_{I}+p\right)}{\left(\Xi_{R}\right)^{k-l+1}} \\
& \times\left(\frac{\left(\rho_{R} \sigma_{e_{1}}^{2}+1\right) m_{1}}{\Omega_{1} \rho_{R}}\right)^{b_{1}}\left(\frac{\Omega_{I}}{m_{I}\left(\rho_{S} \sigma_{e_{R}}^{2}+1\right)}\right)^{p}\left(\frac{\left(\rho_{S} \sigma_{e_{R}}^{2}+1\right)}{\rho_{S}}\right)^{l} \\
& \times \frac{\pi}{P} \sum_{p=0}^{P} \frac{A_{1} \sqrt{1-\theta_{p}^{2}} e^{-\Psi_{1}}}{2 A_{2}+A_{1}\left(1+\theta_{p}\right)}\left(1+\frac{\Omega_{I} \Xi_{R}\left(1+\theta_{p}\right)}{A_{2} m_{I} \rho_{S}\left(1-\theta_{p}\right)}\right)^{-m_{I}-p}\left(\frac{\left(1+\theta_{p}\right)}{A_{2}\left(1-\theta_{p}\right)}\right)^{b_{1}+l}
\end{aligned}
$$

Proof: See Appendix D

3.6 Ergodic capacity $D_{2}$

Similarly, the ergodic capacity of $x_{2}$ is written as

$$
R_{x_{2}}=\frac{1}{2 \ln (2)} \int_{0}^{\infty} \frac{1-F_{Q_{2}}(y)}{1+y} d y,
$$

where $Q_{2}=\min \left(\Gamma_{R \rightarrow x_{2}}, \Gamma_{D_{1} \rightarrow x_{2}}\right)$.

Proposition 5: The closed-form ergodic capacity of $x_{1}$ is given as (29), where $\Psi_{2}=\frac{\Xi_{R}\left(\rho_{S} \sigma_{e_{R}}^{2}+1\right)}{\rho_{R} A_{2}}+\frac{m_{2}\left(\rho_{R} \sigma_{e_{2}}^{2}+1\right)}{\Omega_{2} \rho_{R} A_{2}}$ and $G_{1,[1: 1], 0,[1: 1]}^{1,1,1,1,1}[$ denotes the Meijer-G function with two variables [42].

$$
\begin{aligned}
& R_{x_{2}}=\frac{\alpha_{R}}{2 \ln (2)} \sum_{k=0}^{m_{R}-1} \sum_{l=0}^{k} \sum_{p=0}^{l} \sum_{b_{2}=0}^{m_{2}-1}\left(\begin{array}{c}
l \\
p
\end{array}\right) \frac{k ! \xi(k)\left(\Xi_{R}\right)^{-k+l-1}}{b_{2} ! l ! \Gamma\left(m_{I}\right)} \\
& \times\left(\frac{m_{2}\left(\rho_{R} \sigma_{e_{2}}^{2}+1\right)}{\Omega_{2} \rho_{R} A_{2}}\right)^{b_{2}}\left(\frac{\left(\rho_{S} \sigma_{e_{R}}^{2}+1\right)}{\rho_{R} A_{2}}\right)^{l}\left(\frac{\Omega_{I}}{m_{I}\left(\rho_{S} \sigma_{e_{R}}^{2}+1\right)}\right)^{p} \\
& \times G_{1,[1: 1], 0,[1: 1]}^{1,1,1,1,1}\left[\begin{array}{c|c}
\frac{\Omega_{I} \Xi_{R}}{m_{I} \rho_{R} A_{2} \Psi_{2}} & 1-l+b_{2} \\
\frac{1}{\Psi_{2}} & - \\
& 0,0
\end{array}\right]
\end{aligned}
$$

Proof: See Appendix E

\section{Simulation results and discussion}

In this section, we set $\rho_{C}=1 \mathrm{~dB}, \rho=\rho_{S}=\rho_{R}$ and the main parameters given in Table 3 . The Shadowed-Rician fading parameters for the satellite link 
Table 3 SYSTEM PARAMETERS.

\begin{tabular}{|l|l|}
\hline System Parameters & Values \\
\hline Monte Carlo simulation & $10^{6}$ iterations \\
\hline Power allocation & $A_{1}=0.7$ and $A_{2}=0.3$ \\
\hline Target rate & $R_{1}=0.3$ and $R_{2}=0.5$ bit per channel use \\
\hline Mean square error of channel & $\sigma^{2}=\sigma_{e_{R}}^{2}=\sigma_{e_{1}}^{2}=\sigma_{e_{2}}^{2}=0.001$ \\
\hline Fading severity & $m_{1}=m_{2}=2$ \\
\hline Average power & $\Omega_{1}=\Omega_{2}=1$ \\
\hline
\end{tabular}

Table 4 SATELLITE LINK PARAMETERS.

\begin{tabular}{|l|l|l|l|}
\hline Shadowing & $m_{R}$ & $b_{R}$ & $\Omega_{R}$ \\
\hline Heavy shadowing (HS) & 1 & 0.063 & 0.0007 \\
\hline Average shadowing (AS) & 5 & 0.251 & 0.279 \\
\hline
\end{tabular}

Table 5 INTERFERENCE LINK PARAMETERS.

\begin{tabular}{|l|l|l|l|l|l|}
\hline $\mathrm{N}$ & 1 & 2 & 3 & 4 & 5 \\
\hline$\Omega_{C n}$ & 1 & 2.5 & 2.5 & 3.2 & 3.5 \\
\hline$m_{C n}$ & 1 & 2 & 2.5 & 3 & 3.5 \\
\hline$\Omega_{I}$ & 1 & 3.5 & 6 & 9.2 & 12.7 \\
\hline$m_{I}$ & 1 & 2.9697 & 5.4340 & 8.4317 & 11.9136 \\
\hline
\end{tabular}

are taken from [40] and shown in Table 4. Additionally, the interference channels parameters were set and calculated according to the respective analytical curves in [37] and are shown in Table 5.

Figure ?? shows the outage performance versus the $\rho(\mathrm{dB})$ for different shadowing satellite links. We can observe that the performance of the system under AS is superior to the system under HS. We can also observe the difference in performance of the NOMA and OMA systems. In the OMA system, the gap between the two curves shows that with increased SNR, system performance increases similarly to the NOMA system. The authenticity of the derived expressions is also evident from the strict match of the Monte-Carlo simulations with the analytical simulations.

Figure ?? indicates the outage probability versus $\rho$ under HS by varying $\sigma^{2}$. An increase in the value of $\sigma^{2}$ shows a reduction in the performance of the user, and for the lowest value of $\sigma^{2}$, both users demonstrate better performance. As the SNR increases, the performance of both users continues to increase, while in similar conditions in Figure ??, we varied the number of interfering links for both users by keeping $\sigma^{2}$ constant. The simulation shows that with aa greater number of interference links, the performance of both users decreases. However, in all the links, the curves for each user meet at a saturated point at high SNR. We conclude that in the high SNR region, interference links do not have a great effect on user performance.

Figure ?? shows the simulation for outage performance versus $\rho(\mathrm{dB})$ with the different satellite links as in Figure ??. The ergodic capacity rates of the message at $D_{1}$ are almost similar in both HS and AS modes, but for messaging at $D_{2}$, the gap between the curves of ergodic capacity in both modes is 
comparatively very high. With a simultaneous increase in the SNR, the gap increase is unlike $D_{1}$.

Figure ?? shows the simulation of ergodic capacity versus $\rho(\mathrm{dB})$ under HS by varying $\sigma^{2}$. As we increase the value of $\sigma^{2}$, the gap between the curves increases simultaneously at high SNR values. Figure ?? shows the ergodic capacity versus $\rho(\mathrm{dB})$ varying $N$ with satellite link under HS. Although gaps between the curves are evident at medium SNR values, the curves for both the users meet at a single point at higher SNRs, suggesting that at higher SNRs, a greater number of interference links does not show much differential effect on ergodic capacity rates.

\section{Conclusion}

We described the use of the NOMA technique for communication between a satellite to a relay and the relay to users. We investigated performance of the system in terms of outage probability and ergodic capacity under the effect of CCI at the relay. The performance gap between two destinations can be explained by the differences in power level allocated to the destinations. We also derived the closed-form expressions for outage probability and ergodic capacity at both users. We observed that with an increase in the channel error, both the outage probability and ergodic capacity of the users were significantly affected. The effect of CCI on both outage probability and ergodic capacity is more prominent when the SNR falls in range of 20 to $30 \mathrm{~dB}$, whereas a greater number of interference links shows little effect in the high SNR region. We also simulated and compared system performance under AS and HS modes for both outage probability and ergodic capacity.

\section{Appendix A}

Using (3), $B_{1}$ is written as

$$
\begin{aligned}
& B_{1}=\operatorname{Pr}\left(\frac{\rho_{S} A_{1}\left|\hat{h}_{R}\right|^{2}}{\rho_{S} A_{2}\left|\hat{h}_{R}\right|^{2}+\gamma_{C}+\rho_{S} \sigma_{e_{R}}^{2}+1}>\gamma_{1}\right) \\
& =\operatorname{Pr}\left(\left|\hat{h}_{R}\right|^{2}>\left(\gamma_{C}+\rho_{S} \sigma_{R}^{2}+1\right) \phi_{1}\right),
\end{aligned}
$$

where $\phi_{1}=\frac{\gamma_{1}}{\left(A_{1}-A_{2} \gamma_{1}\right) \rho} . B_{1}$ can therefore be expressed as

$$
B_{1}=\int_{0}^{\infty} f_{\gamma_{C}}(x)\left(1-F_{\left|\hat{h}_{R}\right|^{2}}\left(\left(x+\rho_{S} \sigma_{R}^{2}+1\right) \phi_{1}\right)\right) d x .
$$


Substituting (11) and (12) into (31), $B_{1}$ is rewritten as

$$
\begin{aligned}
& B_{1}=\alpha_{R} \sum_{k=0}^{m_{R}-1} \sum_{l=0}^{k} \frac{\xi(k) k !\left(\phi_{1}\right)^{l} e^{-\Xi_{R} \phi_{1}\left(\rho_{S} \sigma_{R}^{2}+1\right)}}{l ! \Gamma\left(m_{I}\right)\left(\Xi_{R}\right)^{k-l+1}}\left(\frac{m_{I}}{\Omega_{I}}\right)^{m_{I}} \\
& \times \int_{0}^{\infty} x^{m_{I}-1}\left(x+\rho_{S} \sigma_{R}^{2}+1\right)^{l} e^{-\left(\frac{m_{I}+\Omega_{I} \Xi_{R} \phi_{1}}{\Omega_{I}}\right) x} d x .
\end{aligned}
$$

Based on [44, Eq. 1.111] and [44, Eq. 3.381.4], the closed-form of $B_{1}$ is obtained as

$$
\begin{aligned}
& B_{1}=\alpha_{R} \sum_{k=0}^{m_{R}-1} \sum_{l=0}^{k} \sum_{p=0}^{l}\left(\begin{array}{l}
l \\
p
\end{array}\right) \frac{\xi(k) k ! \Gamma\left(m_{I}+p\right)}{l ! \Gamma\left(m_{I}\right)\left(\Xi_{R}\right)^{k-l+1}} e^{-\Xi_{R} \phi_{1}\left(\rho_{S} \sigma_{R}^{2}+1\right)} \\
& \times\left(\phi_{1}\left(\rho_{S} \sigma_{R}^{2}+1\right)\right)^{l}\left(1+\frac{\Omega_{I} \Xi_{R} \phi_{1}}{m_{I}}\right)^{-m_{I}-p}\left(\frac{\Omega_{I}}{m_{I}\left(\rho_{S} \sigma_{R}^{2}+1\right)}\right)^{p} .
\end{aligned}
$$

This completes the proof.

\section{Appendix B}

Let us denote the first and second terms of (21) as $C_{1}$ and $C_{2}$, respectively. Using (4), $C_{1}$ can then be written as

$$
\begin{aligned}
& C_{1}=\operatorname{Pr}\left(\frac{\rho_{S} A_{2}\left|\hat{h}_{R}\right|^{2}}{\gamma_{C}+\rho_{S} \sigma_{e_{R}}^{2}+1}>\gamma_{2}\right) \\
& =\operatorname{Pr}\left(\left|\hat{h}_{R}\right|^{2}>\psi_{1}\left(\gamma_{C}+\rho_{S} \sigma_{e_{R}}^{2}+1\right)\right) \\
& =\int_{0}^{\infty} f_{\gamma_{C}}(x) F_{\left|\hat{h}_{R}\right|^{2}}\left(\left(x+\rho_{S} \sigma_{e_{R}}^{2}+1\right) \psi_{1}\right) d x,
\end{aligned}
$$

where $\psi_{1}=\frac{\gamma_{2}}{\rho_{S} A_{2}}$. As in Proposition 1, we obtain $C_{1}$ as

$$
\begin{aligned}
& C_{1}=\alpha_{R} \sum_{k=0}^{m_{R}-1} \sum_{l=0}^{k} \sum_{p=0}^{l}\left(\begin{array}{c}
l \\
p
\end{array}\right) \frac{k ! \xi(k) \Gamma\left(m_{I}+p\right)}{l ! \Gamma\left(m_{I}\right)\left(\Xi_{R}\right)^{k-l+1}} e^{-\Xi_{R} \psi_{1}\left(\rho_{S} \sigma_{e_{R}}^{2}+1\right)} \\
& \times\left(\psi_{1}\left(\rho_{S} \sigma_{e_{R}}^{2}+1\right)\right)^{l}\left(1+\frac{\Omega_{I} \Xi_{R} \psi_{1}}{m_{I}}\right)^{-m_{I}-p}\left(\frac{\Omega_{I}}{m_{I}\left(\rho_{S} \sigma_{e_{R}}^{2}+1\right)}\right)^{p} .
\end{aligned}
$$


Next, $C_{2}$ is calculated as

$$
\begin{aligned}
& B_{2}=\operatorname{Pr}\left(\frac{\rho_{R} A_{2}\left|\hat{h}_{2}\right|^{2}}{\rho_{R} \sigma e_{2}{ }^{2}+1}>\gamma_{2}\right) \\
& =\operatorname{Pr}\left(\left|\hat{h}_{2}\right|^{2}>\psi_{2}\right) \\
& =\sum_{b_{2}=0}^{m_{2}-1} \frac{e^{-\frac{m_{2} \psi_{2}}{\Omega_{2}}}}{b_{2} !}\left(\frac{m_{2} \psi_{2}}{\Omega_{2}}\right)^{b_{2}},
\end{aligned}
$$

where $\psi_{2}=\frac{\left(\rho_{R} \sigma_{e_{2}}^{2}+1\right) \gamma_{2}}{\rho_{R} A_{2}}$.

Using (35) and (36), the closed-form outage probability of $D_{2}$ is obtained as (22).

This completes the proof.

\section{Appendix C}

In the high SNR region, the CDF of $\left|\hat{h}_{R}\right|^{2}$ and $\left|\hat{h}_{i}\right|^{2}$ are respectively given $\mathrm{s}$

$$
F_{\left|h_{R}\right|^{2}}^{\infty}(x)=\alpha_{R} x
$$

and

$$
F_{\left|h_{i}\right|^{2}}(x)=\frac{1}{\Gamma\left(m_{i}+1\right)}\left(\frac{m_{i}}{\Omega_{i}} x\right)^{m_{i}} .
$$

Next, the asymptotic outage probability of $D_{1}$ is calculated as

$$
P_{D_{1}}^{\infty}=1-B_{1}^{\infty} \times B_{2}^{\infty}
$$

Then, $B_{1}^{\infty}$ is expressed by

$$
\begin{aligned}
& B_{1}^{\infty}=1-\operatorname{Pr}\left(\left|\hat{h}_{R}\right|^{2}<\left(\gamma_{C}+\rho_{S} \sigma_{e_{R}}^{2}+1\right) \phi_{1}\right) \\
& =1-\int_{0}^{\infty} f_{\gamma_{C}}(x) F_{\left|\hat{h}_{R}\right|^{2}}^{\infty}\left(\left(x+\rho_{S} \sigma_{e_{R}}^{2}+1\right) \phi_{1}\right) .
\end{aligned}
$$

Using (37) and (14), we can rewrite $B_{1}^{\infty}$ as

$$
\begin{aligned}
& B_{1}^{\infty}=1-\left(\frac{m_{I}}{\Omega_{I}}\right)^{m_{I}} \frac{\alpha_{R} \phi_{1}}{\Gamma\left(m_{I}\right)} \int_{0}^{\infty} \frac{x^{m_{I}-1} e^{-\left(\frac{m_{I}}{\Omega_{I}}\right) x}}{\left(x+\rho_{S} \sigma_{e_{R}}^{2}+1\right)^{-1}} \\
& =1-\left(\frac{m_{I}}{\Omega_{I}}\right)^{m_{I}} \frac{\alpha_{R} \varphi_{1}}{\Gamma\left(m_{I}\right)}\left(\int_{0}^{\infty} x^{m_{I}} e^{-\left(\frac{m_{I}}{\Omega_{I}}\right) x} d x\right. \\
& \left.+\left(\rho_{S} \sigma_{e_{R}}^{2}+1\right) \int_{0}^{\infty} x^{m_{I}-1} e^{-\left(\frac{m_{I}}{\Omega_{I}}\right) x} d x\right) .
\end{aligned}
$$


$B_{1}^{\infty}$ is thus obtained by

$$
B_{1}^{\infty}=1-\alpha_{R} \phi_{1}\left(\frac{\left(m_{I}\right) !}{\Gamma\left(m_{I}\right)}\left(\frac{\Omega_{I}}{m_{I}}\right)+\left(\rho_{S} \sigma_{e_{R}}^{2}+1\right)\right) .
$$

Then, the term $B_{2}^{\infty}$ can be calculated by

$$
\begin{aligned}
& B_{2}^{\infty}=\operatorname{Pr}\left(\left|\hat{h}_{1}\right|^{2}>\phi_{2}\right) \\
& =1-F_{\left|\hat{h}_{1}\right|^{2}}^{\infty}\left(\phi_{2}\right) \\
& =1-\frac{1}{\Gamma\left(m_{1}+1\right)}\left(\frac{m_{1} \phi_{2}}{\Omega_{1}}\right)^{m_{1}} .
\end{aligned}
$$

Substituting (42) and (43) into (39), we obtain (24).

This completes the proof.

\section{Appendix D}

First, the CDF of $Q_{1}$ is expressed as

$$
\begin{aligned}
& F_{Q_{1}}(x)=\alpha_{R} \sum_{k=0}^{m_{R}-1} \sum_{l=0}^{k} \sum_{p=0}^{l} \sum_{b_{1}=0}^{m_{1}-1}\left(\begin{array}{l}
l \\
p
\end{array}\right) \frac{\xi(k) k ! \Gamma\left(m_{I}+p\right)}{b_{1} ! l ! \Gamma\left(m_{I}\right)\left(\Xi_{R}\right)^{k-l+1}} \\
& \times\left(\frac{\left(\rho_{R} \sigma_{e_{1}}^{2}+1\right) m_{1} x}{\left(A_{1}-A_{2} x\right) \Omega_{1} \rho_{R}}\right)^{b_{1}}\left(1+\frac{\Omega_{I} \Xi_{R} x}{\left(A_{1}-A_{2} x\right) m_{I} \rho_{S}}\right)^{-m_{I}-p}\left(\frac{\Omega_{I}}{m_{I}\left(\rho_{S} \sigma_{e_{R}}^{2}+1\right)}\right)^{p} \\
& \times e^{-\frac{x\left(\rho_{S} \sigma_{e_{R}}^{2}+1\right) \Xi_{R}}{\left(A_{1}-A_{2} x\right) \rho_{S}}-\frac{\left(\rho_{R} \sigma_{e_{1}}^{2}+1\right) m_{1} x}{\left(A_{1}-A_{2} x\right) \Omega_{1} \rho_{R}}}\left(\frac{x\left(\rho_{S} \sigma_{e_{R}}^{2}+1\right)}{\left(A_{1}-A_{2} x\right) \rho_{S}}\right)^{l} .
\end{aligned}
$$

Substituting (44) into (26), we obtain $R_{x_{1}}$ as

$$
\begin{aligned}
& R_{x_{1}}=\frac{\alpha_{R}}{2 \ln (2)} \sum_{k=0}^{m_{R}-1} \sum_{l=0}^{k} \sum_{p=0}^{l} \sum_{b_{1}=0}^{m_{1}-1}\left(\begin{array}{l}
l \\
p
\end{array}\right) \frac{\xi(k) k ! \Gamma\left(m_{I}+p\right)}{b_{1} ! l ! \Gamma\left(m_{I}\right)\left(\Xi_{R}\right)^{k-l+1}} \\
& \times\left(\frac{\left(\rho_{R} \sigma_{e_{1}}^{2}+1\right) m_{1}}{\Omega_{1} \rho_{R}}\right)^{b_{1}}\left(\frac{\Omega_{I}}{m_{I}\left(\rho_{S} \sigma_{e_{R}}^{2}+1\right)}\right)^{p}\left(\frac{\left(\rho_{S} \sigma_{e_{R}}^{2}+1\right)}{\rho_{S}}\right)^{l} \\
& \times \int_{0}^{A_{1} / A_{2}} \frac{1}{1+x}\left(1+\frac{\Omega_{I} \Xi_{R} x}{\left(A_{1}-A_{2} x\right) m_{I} \rho_{S}}\right)^{-m_{I}-p}\left(\frac{x}{\left(A_{1}-A_{2} x\right)}\right)^{b_{1}+l} \\
& \times e^{-\frac{\left(\rho_{S} \sigma_{e_{R}}^{2}+1\right) \Xi_{R} x}{\left(A_{1}-A_{2} x\right) \rho_{S}}-\frac{\left(\rho_{R} \sigma_{e_{1}}^{2}+1\right) m_{1} x}{\left(A_{1}-A_{2} x\right) \Omega_{1} \rho_{R}}} .
\end{aligned}
$$

Using the Gaussian-Chebyshev property and denoting $\theta_{p}=\cos \left(\frac{2 p-1}{2 P} \pi\right)$, the closed-form ergodic capacity of $x_{1}(27)$ can be obtained.

This completes the proof. 


\section{Appendix E}

Similarly, the CDF of $Q_{2}$ is expressed as

$$
\begin{aligned}
& F_{Q_{2}}(y)=1-\alpha_{R} \sum_{k=0}^{m_{R}-1} \sum_{l=0}^{k} \sum_{p=0}^{l} \sum_{b_{2}=0}^{m_{2}-1}\left(\begin{array}{l}
l \\
p
\end{array}\right) \frac{k ! \xi(k) \Gamma\left(m_{I}+p\right)}{b_{2} ! l ! \Gamma\left(m_{I}\right)\left(\Xi_{R}\right)^{k-l+1}} \\
& \times\left(\frac{m_{2}\left(\rho_{R} \sigma_{e_{2}}^{2}+1\right)}{\Omega_{2} \rho_{R} A_{2}}\right)^{b_{2}}\left(\frac{\left(\rho_{S} \sigma_{e_{R}}^{2}+1\right)}{\rho_{R} A_{2}}\right)^{l}\left(\frac{\Omega_{I}}{m_{I}\left(\rho_{S} \sigma_{e_{R}}^{2}+1\right)}\right)^{p} \\
& \times \frac{y^{l+b_{2}}}{\left(1+\frac{\Omega_{I} \Xi_{R}}{m_{I} \rho_{R} A_{2}} y\right)^{m_{I}+p}} e^{-\Psi_{2} y},
\end{aligned}
$$

where $\Psi_{2}=\frac{\Xi_{R}\left(\rho_{S} \sigma_{e_{R}}^{2}+1\right)}{\rho_{R} A_{2}}+\frac{m_{2}\left(\rho_{R} \sigma_{e_{2}}^{2}+1\right)}{\Omega_{2} \rho_{R} A_{2}}$. Next, we can calculate $R_{x_{2}}$ as

$$
\begin{aligned}
& R_{x_{2}}=\frac{\alpha_{R}}{2 \ln (2)} \sum_{k=0}^{m_{R}-1} \sum_{l=0}^{k} \sum_{p=0}^{l} \sum_{b_{2}=0}^{m_{2}-1}\left(\begin{array}{l}
l \\
p
\end{array}\right) \frac{k ! \xi(k) \Gamma\left(m_{I}+p\right)}{b_{2} ! l ! \Gamma\left(m_{I}\right)\left(\Xi_{R}\right)^{k-l+1}} \\
& \times\left(\frac{m_{2}\left(\rho_{R} \sigma_{e_{2}}^{2}+1\right)}{\Omega_{2} \rho_{R} A_{2}}\right)^{b_{2}}\left(\frac{\left(\rho_{S} \sigma_{e_{R}}^{2}+1\right)}{\rho_{R} A_{2}}\right)^{l}\left(\frac{\Omega_{I}}{m_{I}\left(\rho_{S} \sigma_{e_{R}}^{2}+1\right)}\right)^{p} \\
& \times \int_{0}^{\infty} \frac{y^{l+b_{2}} e^{-\Psi_{2} y}}{(1+y)\left(1+\frac{\Omega_{I} \Xi_{R}}{m_{I} \rho_{R} A_{2}} y\right)^{m_{I}+p}} .
\end{aligned}
$$

Using [41], we have

$$
(1+a x)^{-b}=\frac{1}{\Gamma(b)} G_{1,1}^{1,1}\left[\begin{array}{l|l}
a x & \begin{array}{c}
1-b \\
0
\end{array}
\end{array}\right]
$$

where $G_{1,1}^{1,1}[.,$.$] is the Meijer G-function [44]. Substituting (48) into (47), R_{x_{2}}$ is rewritten as

$$
\begin{aligned}
& R_{x_{2}}=\frac{\alpha_{R}}{2 \ln (2)} \sum_{k=0}^{m_{R}-1} \sum_{l=0}^{k} \sum_{p=0}^{l} \sum_{b_{2}=0}^{m_{2}-1}\left(\begin{array}{c}
l \\
p
\end{array}\right) \frac{k ! \xi(k)\left(\Xi_{R}\right)^{l-k-1}}{b_{2} ! l ! \Gamma\left(m_{I}\right)} \\
& \times\left(\frac{m_{2}\left(\rho_{R} \sigma_{e_{2}}^{2}+1\right)}{\Omega_{2} \rho_{R} A_{2}}\right)^{b_{2}}\left(\frac{\left(\rho_{S} \sigma_{e_{R}}^{2}+1\right)}{\rho_{R} A_{2}}\right)^{l}\left(\frac{\Omega_{I}}{m_{I}\left(\rho_{S} \sigma_{e_{R}}^{2}+1\right)}\right)^{p} \\
& \times \int_{0}^{\infty} y^{l+b_{2}} e^{-\Psi_{2} y} G_{1,1}^{1,1}\left[x \mid \begin{array}{l}
0 \\
0
\end{array}\right] G_{1,1}^{1,1}\left[\frac{\Omega_{I} \Xi_{R}}{m_{I} \rho_{R} A_{2}} y \mid \begin{array}{c}
1-m_{I}-p \\
0
\end{array}\right]
\end{aligned}
$$

Based on [43, 2.6.2], (10) is obtained.

This completes the proof. 
Abbreviations

NOMA: Non-orthogonal multiple access; CSI: Channel state information; SIC: Successive interference cancellation; CCI: Co-channel interference; HS: Heavy Shadowing; AS: Average Shadowing; SNR: Signal to noise ratio; QoS: Quality of services; 5G: Fifth generation; IoT: Internet of Things; OMA: Orthogonal multiple access; CI: Channel impairments; HSRN: hybrid satellite relay networks; TMCN: Terrestrial mobile communications network; ISTN: Integrated terrestrial satellite networks; AF: Amplify and forward; DF: Decode and forward; HSTAFRN: Hybrid satellite-terrestrial amplify-and-forward relay network; OP: Outage probability; EC: Ergodic capacity; SND: simultaneous non-unique detection; RVs: Random variables; AWGN: Additive white Gaussian noise; LOS: Light of sight; PDF: Probability density function; CDF: Cumulative distribution function.

\section{Author's contributions}

All authors equally contributed to the work. All authors read and approved the final manuscript.

\section{Funding}

The research leading to these results was supported by the Ministry of Education, Youth and Sports of the Czech Republic under the grant SP2021/25 and e-INFRA CZ (ID:90140)

\section{Availability of data and materials}

Please contact the corresponding author for data requests.

\section{Declarations}

\section{Competing interests}

The authors declare that they have no competing interests.

\section{Author details}

${ }^{1}$ Faculty of Electrical Engineering and Computer Science, VSB-Technical University of Ostrava, 17. listopadu 2172/15, 70800 Ostrava, Czech Republic, ${ }^{2}$ Faculty of Electronics Technology, Industrial University of Ho Chi Minh City, 
Vietnam, ${ }^{3}$ Innovation Center, Van Lang University, Ho Chi Minh City, Vietnam, ${ }^{4}$ Department of Computer Science and Information Engineering, College of Information and Electrical Engineering, Asia University, Taichung 41354, Taiwan.

\section{References}

1. G. Giambene, S. Kota, and P. Pillai, "Satellite-5G integration: a network perspective." IEEE Network, vol. 32, no. 5, pp. 25-31, Sept./Oct. 2018.

2. S. K. Sharma, S. Chatzinotas, and P.-D. Arapoglou, Satellite communi-cations in the $5 \mathrm{G}$ era, IET, 2018.

3. E. Lutz, M. Werner, and A. Jahn, Satellite systems for personal andbroadband communications, Springer-Verlag Berlin Heidelberg 2000.

4. Y. Saito, Y. Kishiyama, A. Benjebbour, T. Nakamura, A. Li, and K.Higuchi, "Nonorthogonal multiple access (NOMA) for cellular futureradio access," inProc. IEEE VTC, Dresden, Germany, Jun. 2013.

5. D.-T. Do, M. -S. Van Nguyen, M. Voznak, A. Kwasinski and J. N. de Souza, "Performance Analysis of Clustering Car-Following V2X System with Wireless Power Transfer and Massive Connections," in IEEE Internet of Things Journal, doi: 10.1109/JIOT.2021.3070744..

6. Dinh-Thuan Do and M.-S. Van Nguyen, "Device-to-device transmission modes in NOMA network with and without Wireless Power Transfer," Computer Communications (Elsevier), vol. 139, pp. 67-77, 2019.

7. D.-T. Do, M. -S. V. Nguyen, F. Jameel, R. Jäntti and I. S. Ansari, "Performance Evaluation of Relay-Aided CR-NOMA for Beyond 5G Communications," in IEEE Access, vol. 8, pp. 134838-134855, 2020.

8. Z. Yang, Z. Ding, P. Fan and N. Al-Dhahir, "A general power allocation scheme to guarantee quality of service in downlink and uplink NOMA systems", IEEE Trans. Wireless Commun., vol. 15, no. 11, pp. 7244-7257, Nov. 2016.

9. M. Zeng, A. Yadav, O. A. Dobre, G. I. Tsiropoulos and H. V. Poor, "On the sum rate of MIMO-NOMA and MIMO-OMA systems", IEEE Wireless Commun. Lett., vol. 6, no. 4, pp. 534-537, Aug. 2017.

10. Y. Liu, Z. Ding, M. Elkashlan and H. V. Poor, "Cooperative non-orthogonal multiple access with simultaneous wireless information and power transfer", IEEE J. Sel. Areas Commun., vol. 34, no. 4, pp. 938-953, Apr. 2016.

11. M. Caus, M. A. Vázquez and A. Pérez-Neira, "NOMA and interference limited satellite scenarios", Proc. IEEE ACSSC, pp. 497-501, Nov. 2017.12. X. Yan, H. Xiao, C.-X. Wang and K. An, "On the ergodic capacity of NOMA-based cognitive hybrid satellite terrestrial networks", Proc. ICCC, Oct. 2017.

12. X. Yan, H. Xiao, C.-X. Wang and K. An, "On the ergodic capacity of NOMA-based cognitive hybrid satellite terrestrial networks", Proc. ICCC, Oct. 2017.

13. S. Xie, B. Zhang, D. Guo and B. Zhao, "Performance analysis and power allocation for NOMA-based hybrid satellite-terrestrial relay networks with imperfect channel state information", IEEE Access, vol. 9, pp. 136279-136289, Sep. 2019.

14. S. Xie et al., "Outage analysis and optimization of NOMA-based integrated satelliteterrestrial system with an AF relay", Proc. IEEE Int. Conf. Commun. in China (ICCC), Oct. 2019.

15. X. Zhang et al., "Outage performance of NOMA-based cognitive hybrid satelliteterrestrial overlay networks by amplify-and-forward protocols", IEEE Access, vol. 7, pp. 85372-85381, Jun. 2019.

16. X. Tang, K. An, K. Guo, Y. Huang and S. Wang, "Outage analysis of non-orthogonal multiple access-based integrated satellite-terrestrial relay networks with hardware impairments", IEEE Access, vol. 7, pp. 141258-141267, Jun. 2019.

17. Z. Lin, M. Lin, J.-B. Wang, T. de Cola and J. Wang, "Joint beamforming and power allocation for satellite-terrestrial integrated networks with non-orthogonal multiple access", IEEE J. Sel. Topics Signal Process., vol. 13, no. 3, pp. 657-670, Jun. 2019. 
18. X. Zhu, C. Jiang, L. Kuang, N. Ge and J. Lu, "Non-orthogonal multiple access based integrated terrestrial-satellite networks", IEEE J. Sel. Areas Commun., vol. 35, no. 10, pp. 2253-2267, Oct. 2017.

19. M. Lin, C. Yin, Z. Lin, J.-B. Wang, T. de Cola and J. Ouyang, "Combined beamforming with NOMA for cognitive satellite-terrestrial networks", Proc. IEEE Int. Conf. Commun. (ICC), May 2019.

20. X. Yan, H. Xiao, K. An, G. Zheng and W. Tao, "Hybrid satellite-terrestrial relay networks with cooperative non-orthogonal multiple access", IEEE Commun. Lett., vol. 22, no. 5, pp. 978-981, May 2018.

21. X. Li, M. Liu, D. Deng, J. Li, C. Deng, and Q. Yu, "Power beacon assisted wireless power cooperative relaying using NOMA with hardware impairments and imperfect CSI," International Journal of electronics and Communications, vol. 108, pp. 275-286, 2019.

22. S. Xie, B. Zhang, D. Guo, and B. Zhao, "Performance analysis and power allocation for NOMA-Based hybird satellite-terrestrial relay networks with imperfect channelstateiinformation," IEEE Access, vol. 7, pp. 136279- 136289, 2019.

23. K. An et al., "Performance analysis of multi-antenna hybrid satellite terrestrial relay networks in the presence of interference," IEEE Trans. Commun., vol. 63, no. 11, pp 4390-4404, Nov. 2015.

24. V. Bankey and P. K. Upadhyay, "Ergodic Capacity of Multiuser Hybrid SatelliteTerrestrial Fixed-Gain AF Relay Networks With CCI and Outdated CSI," in IEEE Transactions on Vehicular Technology, vol. 67, no. 5, pp. 4666-4671, May 2018

25. J. Hussein, S. Ikki, S. Boussakta, and C. Tsimenidis, "Performance analysis of opportunistic scheduling in dual-hop multi-user underlay cognitive network in the presence of co-channel interference," IEEE Trans. Veh. Technol., vol. 65, no. 10, pp. 8163-8176, Oct. 2016.

26. D. B. da Costa, H. Ding, M. D. Yacoub, and J. Ge, "Two-way relaying in interferencelimited AF cooperative networks over Nakagamim fading," IEEE Trans. Veh. Technol., vol. 61 , no. 8, pp. 3766-3771, Oct. 2012.

27. P. K. Upadhyay and P. K. Sharma, "Multiuser hybrid satellite-terrestrial relay networks with co-channel interference and feedback latency," in Proc. Eur. Conf. Netw. Commun. Athens, Greece, Jun. 2016, pp. 174-178.

28. K. Guo, Z. Ji, B. Yang, and X. Wang, "NOMA-based integrated satellite-terrestrial multi-relay networks with hardware impairments and partial relay selection scheme," in Proc. IEEE 19th International Conference on Communication Technology, 2019, pp. 10991104.

29. V. Bankey and P. K. Upadhyay, "Physical Layer Security of Multiuser Multirelay Hybrid Satellite-Terrestrial Relay Networks," IEEE Trans. Veh. Technol., vol. 68, no. 3, pp. 24882501, March 2019

30. S. Xie, B. Zhang, D. Guo and B. Zhao, "Performance Analysis and Power Allocation for NOMA-Based Hybrid Satellite-Terrestrial Relay Networks With Imperfect Channel State Information," IEEE Access, vol. 7, pp. 136279-136289, 2019.

31. X. Li et al., "A Unified Framework for HS-UAV NOMA Networks: Performance Analysis and Location Optimization," IEEE Access, vol. 8, pp. 13329-13340, 2020.

32. Arti M. K. and M. R. Bhatnagar, "Beamforming and combining in hybrid satelliteterrestrial cooperative systems," IEEE Commun. Lett., vol. 18, no. 3, pp. 483-486, Mar. 2014.

33. G. Alfano and A. De Maio, "Sum of squared Shadowed-Rice random variables and its application to communication systems performance prediction," IEEE Trans. Wireless Commun., vol. 6, no. 10, pp. 3540-3545, Oct. 2007.

34. D. Do, A. Le and B. M. Lee, "NOMA in Cooperative Underlay Cognitive Radio Networks Under Imperfect SIC," IEEE Access, vol. 8, pp. 86180-86195, 2020.

35. D. B. da Costa, H. Ding, and J. Ge, "Interference-limited relaying transmissions in dual-hop cooperative networks over Nakagami-m fading," IEEE Commun. Lett., vol. 15, no. 5, pp. 503-505, May 2011.

36. D. B. da Costa and M. D. Yacoub, "Outage performance of two hop AF relaying systems with co-channel interferers over Nakagami-m fading," IEEE Commun. Lett., vol. 15, no. 9, pp. 980-982, Sep 2011. 
37. V. Bankey and P. K. Upadhyay, "Ergodic Capacity of Multiuser Hybrid SatelliteTerrestrial Fixed-Gain AF Relay Networks With CCI and Outdated CSI," IEEE Trans. Veh. Technol., vol. 67, no. 5, pp. 4666-4671, May 2018.

38. Y. Liu, Z. Ding, M. Elkashlan, and J. Yuan, "Non-orthogonal multiple access in largescale underlay cognitive radio networks," IEEE Trans. Veh. Technol., vol. 65, no. 12, pp. 10 152-10 157, Dec. 2016.

39. X. Yue, Y. Liu, S. Kang, A. Nallanathan and Y. Chen, "Modeling and Analysis of TwoWay Relay Non-Orthogonal Multiple Access Systems," IEEE Trans. Veh. Technol., vol. 66, no. 9, pp. 3784-3796, Sept. 2018.

40. N. I. Miridakis, D. D. Vergados, and A. Michalas, "Dual-hop communication over a satellite relay and shadowed Rician channels," IEEE Trans. Veh. Technol., vol. 64, no. 9, pp. 4031-4040, Sep. 2015.

41. V. S. Adamchik and O. I. Marichev, "The algorithm for calculating integrals of hypergeometric type functions and its realization in reduce system," Proc. Int. Symp. Symbolic and Algebraic Computation (ISSAC), Tokyo, Japan, Aug. 1990, pp. 212-224.

42. R. P. Agrawal, "Certain transformation formulae and Meijer's G function of two variables," Indian J. Pure Appl. Math., vol. 1, no. 4, 1970.

43. A. M. Mathai and R. K. Saxena, The H-function with Applications in Statistics and Other Disciplines. Wiley Eastern, 1978.

44. I. S. Gradshteyn and I. M. Ryzhik, Table of Integrals, Series and Products, 6th ed. New York, NY, USA: Academic Press, 2000.

\section{Figures Title and Legend Section}

Figure 1. System model.

Figure 2. Outage performance vs $\rho(\mathrm{dB})$ with different satellite links.

Figure 3. Outage performance vs $\rho(\mathrm{dB})$ varying $\sigma^{2}$ with the satellite link under HS.

Figure 4. Outage performance vs $\rho(\mathrm{dB})$ varying $N$ with the satellite link under HS.

Figure 5. Ergodic capacity vs $\rho(\mathrm{dB})$ with different satellite links.

Figure 6 . Ergodic capacity vs $\rho(\mathrm{dB})$ varying $\sigma^{2}$ with the satellite link under HS.

Figure 7. Ergodic capacity vs $\rho(\mathrm{dB})$ varying $N$ with the satellite link under HS. 


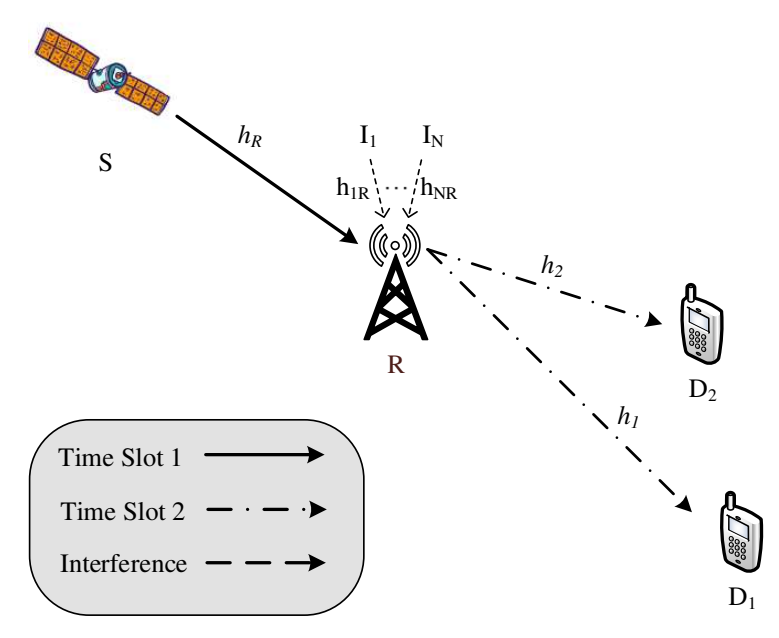

Figure 1 
$N$
0
0
50
.5

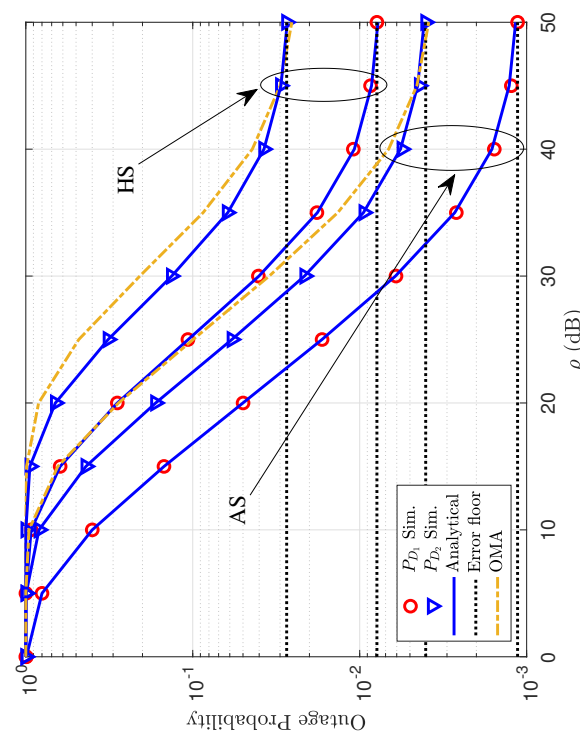




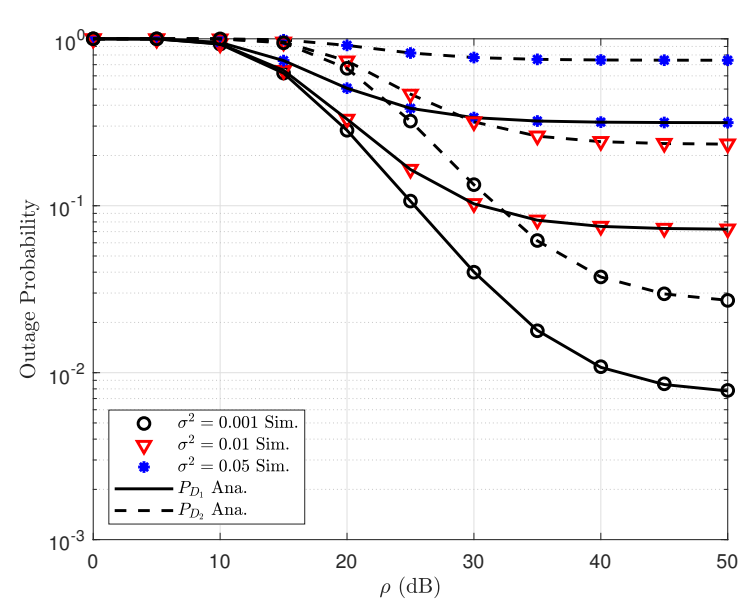

Figure 3 


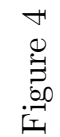

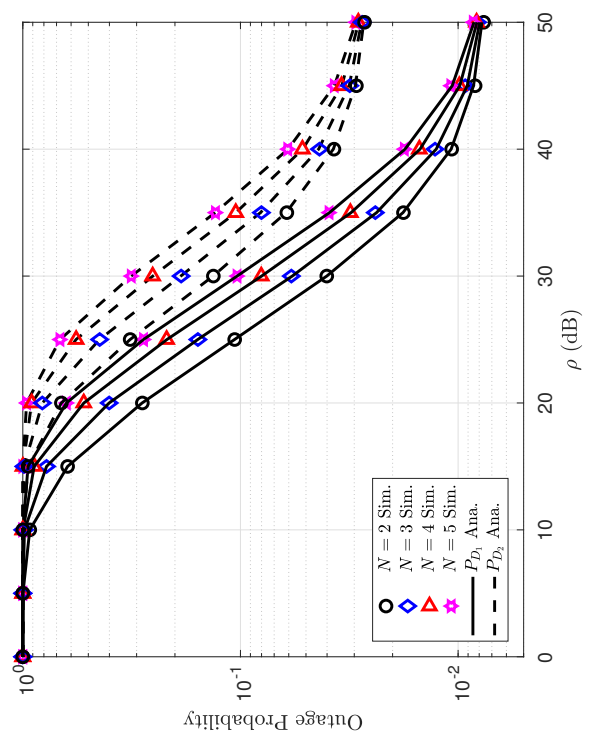




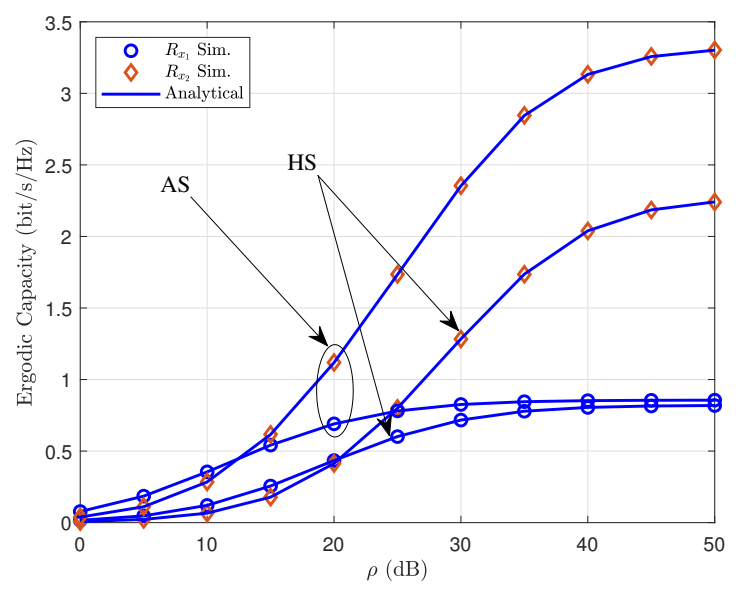

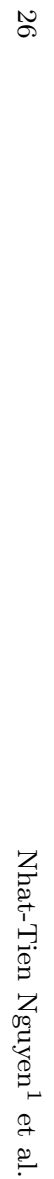

Figure 5 
0
0
$\vdots$
6
.1
1

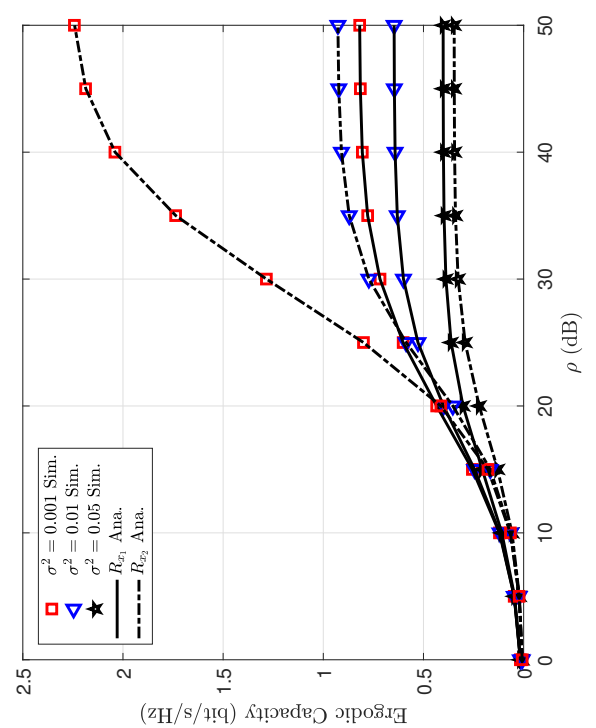




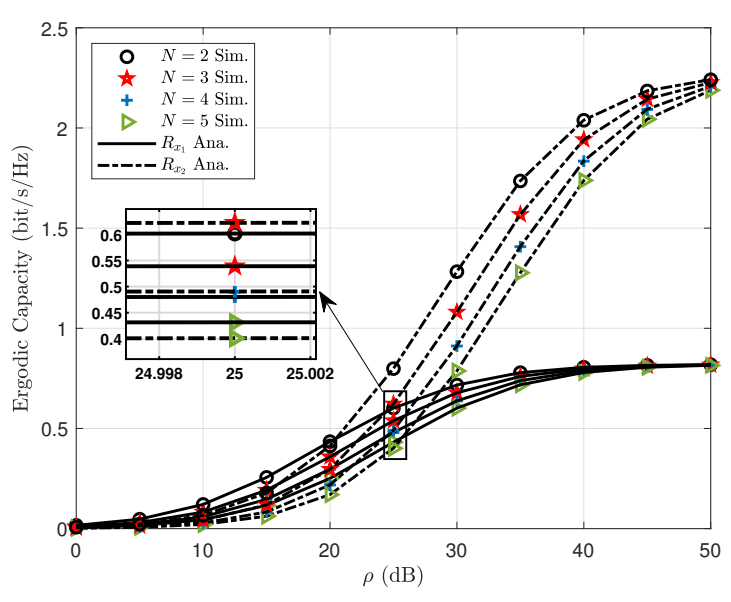

Figure 7 